\title{
Calidad total y comunicaciones
}

\author{
Roberto RAMÍREZ
}

La calidad es hoy más importante que nunca; sin embargo, en algunas organizaciones se presenta como una palabra extraña. Los clientes de hoy no sólo aprecian la calidad sino que la esperan y, de no obtenerla, se van a otra parte; por este motivo es que la existencia de una organización depende mucho de su dedicación a la calidad, de su habilidad para satisfacer las necesidades y de las expectativas cambiantes de sus clientes.

Cada experto y cada organización puede tener su propia definición de calidad total, sin embargo, coinciden en tres aspectos:

- A todos los guían los clientes: internos y externos.

- La calidad total se enfoca hacia el mejoramiento continuo de todos los procesos de la organización. Como resultado, la calidad total es un proceso, no es un programa; un programa acaba en una semana o un año, pero un proceso nunca termina.

- La calidad total involucra a todos en el proceso de mejora, la calidad es un sistema de valores, es una cultura corporativa que requiere de la participación de todos, desde arriba hasta abajo.

\section{MEJORAMIENTO DE LA CALIDAD}

Los autores que trabajan este tema distinguen ciertos pasos a seguir en cuanto al mejoramiento de la calidad. Éstos se pueden resumir en algunas ideas que pasamos a desarrollar. 
1. Serna Humberto. Mercadeo corporativo. Bogotá: Fondo Editorial, 1992
En primer término hay que establecer el estándar de calidad al averiguar las necesidades y expectativas de los clientes; éstas se analizan y se excede el estándar (valor agregado).

Normalmente creemos que conocemos las necesidades del cliente, pero podemos estar equivocados. Para nuestro cliente, por ejemplo, el color puede significar calidad. Lo que realmente cuenta es cómo éste define la calidad.

Hablar con el cliente no siempre es grato, a veces ellos serán muy negativos, pero se necesita oír sus críticas para mantener a los clientes, para satisfacer sus cambiantes necesidades y sus expectativas. Descubrir las necesidades y expectativas de nuestros clientes es importante para la calidad total ${ }^{1}$.

En la organización puede ser que uno no tenga contacto directo o no vea a los clientes y usuarios de los productos y servicios, pero sí tenemos mucho contacto con nuestros clientes internos, es decir con todas las personas a quienes les suministramos información, productos e ideas.

¿Y qué tiene que ver la calidad con servir a nuestros clientes internos?

Si no hacemos un trabajo de calidad, no damos un buen servicio, nuestra organización tiende a la desaparición del mercado como consecuencia de no poder dar un buen servicio a nuestros clientes externos.

\section{LA CALIDAD LA GUÍAN LOS CLIENTES}

La comunicación con los clientes es la forma más utilizada para descubrir sus necesidades. En el caso de los clientes externos, adopta la forma de quejas, devoluciones y reclamos. Cada una de estas insatisfacciones con el producto y/o servicio requiere una atención individual.

La calidad total es guiada por los clientes, debemos indagar sus necesidades o expectativas.

Hagamos preguntas, escuchemos la retroalimentación, estemos abiertos a lo que oigamos, pero recordemos: la retroalimentación no siempre es positiva. Lo que escuchemos nos ayuda a establecer estándares de calidad; lo que cuenta es la percepción de calidad del cliente.

Para asegurar que las necesidades y expectativas de los clientes se encuentren y sean excedidas debemos procurar que el mejoramiento de los procesos sea continuo en nuestra organización, comenzando con las personas, entrenándolas en: 
- habilidades técnicas,

- habilidades de comunicación,

- autoadministración,

- habilidades de equipo.

Sólo las personas bien entrenadas pueden satisfacer las necesidades de los clientes y estar al tanto de los cambios en la tecnología y el mercado.

\section{LAS PERSONAS SON LA BASE MÁS IMPORTANTE DE LA ORGANIZACIÓN}

Mejorar los procesos continuamente es importante para la calidad total. Para mejorar estos procesos basta con indagar con el personal sobre qué mejoras realizar y, al mismo tiempo, establecer metas claras y controles que aseguren la permanencia de estos cambios organizacionales.

Mejorar los procesos requiere de la solución de los problemas, teniendo en consideración que:

- Uno hace mejoras permanentemente a largo plazo. Al resolver problemas críticos no esporádicos, estos problemas deben tener un impacto mayor.

- Los problemas vistos en conjunto nos ayudan a ver el problema total.

- No hay que tomar soluciones rápidas que sólo resolverán el problema temporalmente.

- Se debe dedicar el tiempo necesario a averiguar las causas.

Todos los afectados por el problema deben estar envueltos en el proceso de su solución. Ocasionalmente los problemas de calidad se deben a que los proveedores nos dan servicios deficientes, y cuando eso sucede, los traemos al proceso de solución de problemas, pero la mayoría del tiempo los evitamos comprando sólo a proveedores de primera calidad. Si los proveedores no siguen mejorando sus productos y servicios, es más dificil mejorar los propios. Todos nosotros proveemos de algo a alguien, ya sea dentro o fuera de la organización.

La calidad total es un mejoramiento continuo:

- Mejorando a las personas a través de entrenamiento.

- Mejorando los sistemas.

- Estableciendo un plan de calidad.

- Estableciendo controles.

- Solucionando problemas. 
- Mejorando las relaciones, tanto con los proveedores internos como con los externos.

El mejoramiento continuo nos permite un cambio constante, pero todos, en cualquier nivel, usualmente temen y se resisten al cambio; éste toma tiempo para ser aceptado. Es importante involucrar a todos en el proceso de cambio, tan importante que se considera el tercer punto de la calidad total.

Este involucramiento total requiere de una dedicación también total de arriba hacia abajo. Requiere que los individuos y los equipos trabajen juntos por las mismas metas; quiere decir dar sugerencias y escuchar las ideas de otros. Ningún producto o servicio es responsabilidad sólo de una persona, un departamento o un proceso.

Para que la organización trabaje efectivamente todos deben conocer su función en el movimiento, tomar posesión del trabajo que realizan. El involucramiento total requiere de reconocimiento y éste a su vez muestra a todos que la calidad es prioritaria, contribuyendo a respetar la creatividad, la innovación y el involucramiento. Además, permite conocer los logros de todos aquellos que componen la organización.

En conclusión, la calidad total es trabajar juntos, administrando el propio trabajo, reconociendo los logros de los demás, e involucrando a toda la organización en el proceso de mejora continua.

\section{LA CALIDAD TOTAL IMPLICA UNA ORGANIZACIÓN PARALELA}

Aplicar un programa de calidad total en una organización requiere de una estructura encargada de manejar los procesos de cambio. A continuación se presentan algunas ideas de cómo aplicarlo y de cómo debe estar compuesta dicha estructura.

- Insertar el comité para la calidad total (CCAT) en la empresa: La integra el líder empresarial y sus ejecutivos de más alto nivel en la organización (4 a 7 miembros). Se decide así desarrollar en todo el personal el sano hábito de mejoramiento continuo en todos los procesos de la empresa.

- Difundir los conceptos de calidad total de arriba hacia abajo: charlas, películas, videos tecnológicos, seminarios, invitaciones a expertos, asesores, gerentes que estén aplicando la calidad total. 
Promover la semana de la calidad e integrarse a la red nacional para la calidad.

- Desarrollar e innovar procesos en las empresas impulsados por equipos de mejoramiento de proceso y líderes de proceso. Desarrollar procesos de gestión sepultando el fraccionamiento del trabajo creado por su división: las oficinas separadas, el aislamiento de escritorios, profesiones diferentes, modismos, costumbres, politicas y actitudes separatistas. Proceso y no fraccionamiento.

- Nombrar un facilitador de apoyo en la ejecución de los procesos de calidad total para toda la empresa e involucrar el mejoramiento continuo. Circular por toda la organización promoviendo la calidad total.

- Nombrar un líder de círculos de calidad y planear el proceso de instalación y desarrollo de círculos de calidad hasta obtener voluntariamente una expansión que produzca la verdadera madurez global en la empresa. Multiplicar los círculos de calidad.

- Promover la innovación y creatividad en los procesos de trabajo.

- Crear la "Semana de la Calidad Total en la Empresa”. En esta semana se da énfasis a los valores y estándares de calidad para seguir avanzando en lo que se ha dado en llamar construir la potencialidad de la empresa.

- Consolidar la calidad en todas las áreas y niveles de la organización. Mejorar productos, el servicio, las relaciones, la percepción de las responsabilidades, la tecnología. Dar mayores satisfacciones al cliente, mejorar el prestigio y el liderazgo ${ }^{2}$.

\section{LAS COMUNICACIONES EN LOS PROCESOS DE CALIDAD TOTAL}

Uno de los más importantes procesos de las organizaciones y de los más fáciles de observar es la comunicación de los miembros de la organización entre sí, particularmente en situaciones directas. Muchas veces se ha descrito la comunicación como un sencillo problema de transmitir información de una persona a otra. Sin embargo, todos sabemos que el proceso no es sencillo y que la información es con frecuencia muy variable y compleja.

La comunicación, además de ser un medio para informar al personal, es requisito previo indispensable para mo-
2. Beckhard, Richard. Lo que las empresas deben hacer para lograr una transformación total. San Francisco: Jossey-Bass Publishers, 1992 . 
dificar las actitudes y la conducta, las maneras de trabajar y las relaciones; son elementos necesarios para un cambio fundamental.

Es por ello que en la organización se enfatizan dos tipos de comunicación:

\section{Comunicación pasiva}

Es el tipo de comunicación que más se emplea para informar al personal de una organización acerca de los cambios que se hacen o se piensan hacer. Ésta es una comunicación en un solo sentido (hacia abajo) y no da como resultado el compromiso emocional. Además, las personas o no escuchan o se olvidan lo que se les dice. Los mensajes se tienen que repetir muchas veces.

\section{Comunicación activa}

Es un complemento poderoso de la tradicional comunicación hacia abajo y desempeña una función clave para hacer que ocurra un cambio. Aquí, el proceso está diseñado para que los empleados tomen parte personalmente y para que al recibir los mensajes se pregunten:

¿Cómo me afecta esto a mí, a mi conducta, a mi manera de trabajar, a mis relaciones, etc.?

Además, los empleados se interesan no sólo de manera intelectual sino también emocional. Sin este proceso es probable que no haya cambio ni compromiso. Crear comprensión y sentido de propiedad es parte crucial de una estrategia de comunicación y deben diseñarse como procesos de aprendizaje para la organización.

Con todo esto, lo que se quiere decir es que es de vital importancia la participación activa de todos en la organización, y las comunicaciones tienen un papel preponderante en este contexto. A la mayoria de organizaciones, sin embargo, les resulta muy dificil evitar problemas de comunicación. La estrategia más eficiente y que ha demostrado mayores beneficios radica en formalizar grupos primarios (o círculos de calidad) que sirvan de instrumento para la comunicación tanto ascendente como descendente.

Estos grupos son los encargados de la difusión de las políticas, objetivos y metas de la organización, facilitando igualmente el conocimiento de los productos, servicios y proyectos que se emprendan. A su vez, utilizar estos equipos como herramienta para la participación es un logro difícil de 
alcanzar para las organizaciones. Todo ser humano necesita ser parte de su propio quehacer. La participación en las decisiones crea compromiso y motivación, y genera responsabilidad.

Las empresas deben, de igual modo, establecer sistemas que estimulen la creatividad y la participación voluntaria de los miembros de la organización. La innovación, la calidad total y la excelencia son el resultado del desarrollo de actividades de grupos automotivados que se comprometen con el cambio y la competitividad organizacional.

Las organizaciones hacen un buen trabajo en la comunicación de la información esencial de la calidad a los trabajadores en materias tales como las especificaciones, normas, procedimientos y métodos. Esta información, aunque es necesaria, no es suficiente. También se debe proveer medios que permitan a los empleados comunicar sus puntos de vista e ideas y explicar aquellas actuaciones gerenciales aparentemente antagonistas de la calidad. Para ello se requiere de un liderazgo gerencial integral que logre la vinculación de todos los miembros en los programas de mejora continua.

El liderazgo es como la belleza: dificil de definir pero fácil de reconocer si uno lo ve. Llegar a ser líder no es fácil, pero aprender a dirigir es mucho más fácil de lo que se cree, porque todos tenemos la capacidad de liderazgo. Para ser un líder se requiere de un proceso. No tiene un principio ni un fin, pero sí temas recurrentes como son la necesidad de educación, tanto formal como informal, la necesidad de "desaprender" para aprender, la de reflexionar sobre lo que se aprende, la de tomar riesgos y cometer errores y la necesidad de competencia (ser competente o dominar lo que se va a hacer). Estos temas no sólo se repiten, sino que se entrelazan unos con otros.

No podemos vivir sin líderes. La calidad de nuestra vida depende de la calidad de nuestros líderes. Por ello, existen dos razones por las cuales los líderes son importantes:

- Porque son responsables de la eficiencia de la organización.

- Porque se preocupan por la integridad de nuestras organizaciones.

El contexto nos domina y nos ciega tanto como a un pez el agua: la competencia, el avance tecnológico, la velocidad de las comunicaciones, la aparición de nuevas empresas, etc.

Abraham Zaleznik, profesor de la Universidad de Harvard, dice que hay dos clases de líderes: los que nacen una vez y los que nacen dos veces. Para los que nacen una vez, la transición de hogar y familia hacia la independencia 
es relativamente fácil. Los que nacen dos veces sufren a medida que crecen, se sienten distintos y desarrollan, así, una compleja vida interior. Según Zaleznik, los que nacen dos veces se orientan al interior, están seguros de sí mismos $\mathrm{y}$, como resultado, son realmente carismáticos. Por lo tanto, los que sólo nacen una vez han sido inventados por las circunstancias y los que nacen dos veces se inventaron a sí mismos.

Una persona empieza a ser líder desde el momento en que resuelve por sí misma cómo ser o qué quiere llegar a ser. Lo demás es fácil: estrategias, maneras, formas, modos, estilos de dirección para conseguir lo que busca.

Así, pues, "conócete a ti mismo" significa separar lo que uno es y quiere ser, de lo que el mundo piensa que es y quiere que sea. El autoconocimiento y la autoinvención son procesos de toda la vida.

\section{LOS CÍRCULOS DE CALIDAD}

\section{Historia de los círculos de calidad}

Los aspectos de lo que hoy conocemos como "procesos de calidad total" han variado a lo largo de la historia. Inicialmente se consideraba la calidad como el control estadístico para procesos de producción.

Así, en 1950 la J.U.S.E. (Unión de Científicos e Ingenieros Japoneses) publicó la revista Statistical Quality Control (SQC), que difundía información sobre control de calidad y control total de calidad.

En 1956 se inició en Japón un curso de control de calidad por correspondencia para supervisores, valiéndose de NHK (Radiodifusora Japonesa de Onda Corta); el programa fue muy bien recibido y se vendieron 110.000 ejemplares.

En 1962 la J.U.S.E. publicó la revista Quality Control for the Foreman ( $F Q C)$, dirigida principalmente a los obreros y

3. Ogliastri, Enrique. Gerencia japonesa $y$ círculos de participación. Bogotá: Editorial Norma S.A., 1988 sus supervisores, motivándolos a leer, intercambiar información y procurar el desarrollo mutuo ${ }^{3}$.

Al publicarse esta revista el doctor Kaoru Ishikawa propuso que las actividades de control de calidad tomasen el nombre de círculo de calidad, con el fin básico de estudiar la revista. Consideraba además que todo lo estudiado debía ponerse en práctica en el lugar de trabajo y debía estimularse a las personas para que resolviesen sus problemas laborales. El doctor Ishikawa hizo hincapié en: 
- El voluntarismo: Los círculos han de crearse voluntariamente, no por órdenes de superiores. Se deben comenzar las actividades de los círculos con aquellas personas que deseen participar.

- Autodesarrollo: Los miembros del círculo deben estar dispuestos a estudiar.

- Desarrollo mutuo: Los miembros del círculo deben aspirar a ampliar sus horizontes y a cooperar con otros círculos.

- Participación total: Los círculos deben fijar como meta final la participación plena de todos los empleados del lugar.

En 1962 se creó el primer círculo de calidad. Ese mismo año se organizó la Conferencia Anual de Control de Calidad para supervisores y la Conferencia de Círculos de Calidad. En 1965 ya había 3.700 grupos que practicaban actividades de círculos de calidad. En 1971 se creó el Gran Premio de Círculos de Calidad que se otorga todos los años en el mes de noviembre.

Para que el movimiento perdure es necesario evitar la obligatoriedad y poner énfasis en el voluntarismo y participación. A veces la ruta más lenta es la que lleva al éxito.

En 1983 había 173.953 círculos de calidad, con 1.490.629 miembros inscritos en la revista $F Q C^{4}$. El número de círculos no inscritos debe haber sido diez veces mayor.

\section{Definición de círculo de calidad}

Se denomina círculo de calidad al grupo de personas que se reúnen voluntariamente y en forma periódica para detectar, elegir, analizar y buscar soluciones o mejoras a los problemas que se presentan en su área de trabajo.

\section{Filosofia de los círculos de calidad}

Su filosofia es contribuir con el desarrollo de la empresa a través del respeto y la dignificación del trabajo, del fomento de valores superiores y armónicos, con el pleno desarrollo de la capacidad humana, proyectándola hacia sus posibilidades infinitas.
4. Kasuga, Hermelinda. Círculos de calidad. México: Editorial Grad S.A., 1991. 
4. Objetivos de los círculos de calidad

Sus objetivos son:

- Contribuir con el proceso de mejora continua de la organización.

- Crear un mejor ambiente de trabajo.

- Ejercer las capacidades humanas plenamente.

5. Beneficios de los circulos de calidad

- Potencia la capacidad para identificar y resolver problemas.

- Incrementa el liderazgo personal y la habilidad para trabajar en equipo.

- Facilita la participación en la solución de los problemas.

- Ofrece la oportunidad de aprender más.

- Favorece el reconocimiento.

6. El facilitador en un círculo de calidad

El facilitador es un líder nombrado por la Alta Dirección del programa de calidad instaurado, cuya función principal está encaminada a servir de nexo entre los círculos de calidad y la gerencia de la organización. Sobre la base de esta definición sus roles deben estar encaminados a:

- Renovar permanentemente los roles y compromisos con los círculos de calidad.

- Crear condiciones para que las necesidades de información lleguen oportunamente a los círculos de calidad.

- Motivar y dar el reconocimiento a los avances de los círculos de calidad.

- Retroalimentar el proceso con el feedback correspondiente.

- Facilitar la dinámica e interacción humana de los círculos de calidad con su rol.

- Promover permanentemente el cumplimiento de la metodología del proceso.

- En coordinación con otros facilitadores, promover reuniones entre los líderes para fortalecer el proceso y producir sinergía. 
El líder en un círculo de calidad es un trabajador elegido por sus demás compañeros para que los represente frente al facilitador y la dirección del programa de calidad. Su rol en un proceso de calidad total consiste en:

- Proporcionar un apoyo sincero: El líder debe convencer a los miembros del círculo de que su éxito será posible si se trabaja en equipo. Las reuniones del círculo se deben hacer con regularidad. El líder debe apoyar las iniciativas que el grupo desee tomar.

- Usar técnicas de solución de problemas: Es responsabilidad del líder ver que el grupo continúe usando las técnicas de solución de problemas; sin estas técnicas las reuniones se convierten en sesiones de quejas o en discusiones infructuosas

- Guiar sin dominar: Como líder de un círculo debe recordar que es sólo una voz en el grupo, un miembro más. Debe tener cuidado de no dominar la reunión y no dictar los problemas que debe tratar el grupo. En su papel de líder podrá guiar al grupo pero debe tener mucho cuidado al hacerlo.

- Involucrar a todos los miembros: El líder debe asegurarse de que todos los miembros del círculo tengan la oportunidad de participar en las reuniones. Debe encontrar la forma de que los miembros más tímidos presenten sus problemas y sus ideas de solución a consideración de los demás.

- Mantener informada a la gerencia: El líder debe mantener informada a la gerencia sobre las actividades y el progreso de los círculos, por ejemplo, la publicación de agendas, la invitación a los gerentes a asistir a las reuniones de presentación de resultados, etc.

- Mantener informados a los que no son miembros: Las personas de la sección que no son miembros de círculos deben estar informadas sobre lo que está haciendo el círculo, los problemas que están tratando y las soluciones propuestas que puedan afectarlos. Una de las formas en que se puede informar es realizando foros sobre los círculos en los lugares de trabajo, involucrando a quienes no son miembros en la recopilación de datos, en la elaboración de gráficas u otros datos que pueden ser útiles para el círculo. 
- Cooperar en el seguimiento y medición de los proyectos: Los proyectos deben medirse y es necesario hacerles un seguimiento para asegurar que las soluciones a los problemas sean efectivas. Es responsabilidad del líder, con la asistencia del facilitador, asegurarse de que el grupo verifique el efecto de una solución y de que tome las acciones correctivas necesarias.

- Desarrollar a los miembros del círculo: Los círculos de calidad suponen un concepto de desarrollo personal. Es tarea del lider permitir a los miembros del círculo que asuman la responsabilidad por alguna fase del proyecto o por la conducción de alguna etapa de las reuniones y estimularlos para ello. Se debe permitir a los miembros realizar la función del líder cuando éste se encuentra ausente.

Las estrategias para estimular esta participación voluntaria son muy diversas y dependen de las características de la empresa. Sin embargo, existen algunas que se han consolidado en el entorno. Una de las más importantes es la metodologia utilizada por los círculos de calidad.

Además de los líderes formales de una organización existen los líderes emergentes, quienes proporcionan un apoyo significativo a la empresa. Todos ellos deben cumplir funciones de respaldo al programa de calidad total.

\section{Los miembros}

Son los trabajadores voluntarios que participan en las actividades de un círculo de calidad y cuyo rol consiste en:

- Asistir puntualmente a las reuniones.

- Contribuir al trabajo en equipo.

- Completar oportunamente las tareas asignadas.

- Poner en práctica las técnicas de los círculos de calidad

- Respetar la metodología del proceso.

\section{Actividades de los círculos de calidad}

Los círculos de calidad son los responsables en la organización de:

- Analizar la situación.

- Identificar los problemas.

- Seleccionar el problema a tratar. 
- Analizar el problema.

- Cuantificar el problema (tiempo, dinero, etc.).

- Identificar alternativas de solución.

- Selección de la solución óptima.

- Presentar la propuesta de mejora.

\section{CONCLUSIÓN}

Los programas de calidad total son necesarios hoy más que nunca en las organizaciones debido a lo que representan en términos de desarrollo del personal, de permanencia en el mercado satisfaciendo las necesidades de los clientes, de motivación, involucramiento y mejora continua de la empresa.

Sin embargo, cualquier actividad que se emprenda, sea cual fuere la estructura que se adopte y cualesquiera que sean los programas que se diseñen, no tendrán éxito sin la participación activa de la comunicación como clave para alcanzar la calidad total. 
Bibliografía

Beckhard, Richard [y] Wendy Pritchard 1992

Lo que las empresas deben hacer para lograr una transformación total. San Francisco: Jossey-Bass Publishers.

Kasuga de Yamazaki, Hermelinda 1991

Círculos de calidad. México: Editorial Grad S.A.

Ogliastri, Enrique 1988

Gerencia japonesa y círculos de participación. Bogotá: Editorial Norma S.A.

Schein, Edgar H.

1990

Consultoría de procesos. México: Sistemas Técnicos de Edición S.A.

Serna Gómez, Humberto 1992

Mercadeo corporativo. Bogotá: Fondo Editorial.

Stoner, James A.F. [y] Edward Freeman

1992

Administración. México: Prentice Hall Hispanoamericana S.A. 